\title{
The effect of neighbourhood social ties on migrants' subjective wellbeing in Chinese cities
}

\begin{abstract}
Existing literature on migrants' subjective wellbeing (SWB) in Chinese cities has highlighted the crucial role of social ties, yet the pathways by which social ties influence their SWB remain poorly understood. Using Guangzhou survey data and multilevel linear regressions, this paper examines the extent to and ways in which migrants' social ties with neighbours enhance their SWB, with a particular focus on the distinction between the main effects and buffering effects of their neighbourhood ties. Results from multilevel models reveal that neighbourhood ties enhance migrants' SWB in a direct manner, but no evidence shows that neighbourhood ties lessen the negative impacts of neighbourhood deprivation. Results also illustrate that the association between neighbourhood ties and SWB is stronger for locals than for migrants. This paper contributes to our understanding of migrants' SWB by disentangling the positive effects of their social ties with neighbours and investigating the role of migrants' neighbourhood ties in relation to stress arising from neighbourhood deprivation.
\end{abstract}

Keywords: migrants; subjective wellbeing; neighbourhood ties; neighbourhood deprivation; urban neighbourhoods; China 


\section{Introduction}

Scholars have long noted the role played by neighbourhood social ties in residents' subjective wellbeing (SWB) (Cohen \& Wills, 1985; Helliwell \& Putnam, 2004; Lin \& Ensel, 1989; Ross, Reynolds, \& Geis, 2000). Two models have been proposed to explain the pathways by which residents' connections with neighbours enhance their SWB. The first is the 'main effect' model which indicates that people directly acquire social resources via their relations with neighbours (Cohen \& Wills, 1985; Lin \& Ensel, 1989). The second model focuses on the 'buffering effect', which occurs when the adverse effect of stressors (e.g., work stressors, environmental stressors) on one's mental state is alleviated by social support among neighbours (Cohen \& Wills, 1985; Lin \& Ensel, 1989). The main effect and buffer effect of social ties are not mutually exclusive and can occur simultaneously (Cohen \& Wills, 1985; Lin \& Ensel, 1989). Recent studies on these two effects of neighbourhood ties involve neighbourhood deprivation. For example, Mitra (2010) found that the negative effect of neighbourhood deprivation on residents' SWB was weaker in the presence of supportive and cohesive neighbourhoods. It is only recently that the buffering effect of immigrants' neighbourhood ties on their SWB has received wide attention (Carmel, 2001; Herrero, Fuente, \& Gracia, 2011).

There has been a growing body of literature on the wellbeing of migrants from rural areas and small towns in Chinese cities (Fan, 2002; Li, 2006; F. Wu, 2016; W. Wu, 2004; Zhang \& Tao, 2012). The majority of such work highlighted the role of institutional and economic constraints in lowering migrants' wellbeing (Chan \& Zhang, 
1999; Fan, 2002; Wang \& Fan, 2012; Zhang, 2012). Another stream of literature emphasised the role of migrants' social ties by which migrants acquired labour market opportunities, possibilities for life enhancement, and housing opportunities in host cities (Hui, Yu, \& Ye, 2014; Liu, Li, Liu, \& Chen, 2014; Ma \& Xiang, 1998; Niu, Xu, Liu, Wang, \& Klein, 2016; Yue, Li, Jin, \& Feldman, 2013). Although a plethora of research has documented and analysed the objective conditions of migrants' wellbeing, only a few studies have explored how migrants evaluate their wellbeing in a subjective way (Chen \& Davey, 2008; Jin, Wen, Fan, \& Wang, 2012; Liu, Zhang, Wu, Liu, \& Li, 2017; Wen, Fan, Jin, \& Wang, 2010). Far less attention has been paid to the association between migrants' social ties (especially neighbourhood ties) and their SWB (Jin et al., 2012; Wen \& Wang, 2009), and no attempt has been made to investigate the pathways by which migrants' neighbourhood ties influence their SWB in the Chinese context.

This paper aims to examine the effects of migrants' neighbourhood ties on their SWB in Guangzhou, China, utilising survey data collected in 23 residential communities and multilevel linear regressions. The study particularly focuses on not only how migrants' neighbourhood ties enhance their SWB directly (the main effect), but also how their neighbourhood ties alleviate the negative impacts of neighbourhood deprivation (the buffering effect).

This research contributes to the existing literature in several ways. Conceptually, this study makes the first attempt to disentangle the positive effects of migrants' neighbourhood ties on their SWB in the Chinese context. Empirically, it adopts multilevel models rather than single-level models to accurately measure the estimates 
and isolate effects due to observed and unobserved neighbourhood characteristics. The current study treats the detrimental effect of neighbourhood deprivation as separate from other neighbourhood environmental stressors on migrants' SWB, due to the fact that the index of deprivation is a composite indicator that captures the effect of most other environment stressors (e.g., security and cleanliness).

The remainder of the paper is organised as follows. Section 2 provides a brief review of literature on neighbourhood ties, neighbourhood deprivation, and SWB, as well as their association with migrants in Chinese cities. Section 3 describes data, methods, and the measurement of variables used in this study. Section 4 examines the main effects and buffering effects of migrants' neighbourhood ties on their SWB, while treating local residents as a reference group. Finally, section 5 summarises the main findings of this research and discusses the policy implications as well as research limitations.

\section{Literature review}

\subsection{Neighbourhood ties and SWB}

Neighbourhood ties consist of neighbourly interactions and mutual assistance among neighbours (Wellman \& Wortley, 1990). Previous studies have shown a strong association between social ties and SWB (Cohen \& Wills, 1985; Helliwell \& Putnam, 2004; Lin \& Ensel, 1989; Ross \& Jang, 2000; Sampson, 2003). Cohen and Wills (1985) proposed two models to explain the mechanism by which neighbourhood ties influenced residents' SWB: the main effect model and buffer effect model. The main 
effect model suggested that reciprocal neighbourhood ties increased individuals' SWB in two ways: first, individuals receive instrumental support from their neighbours when seeking jobs, loans, and temporary childcare; second, individuals receive emotional support from their neighbours and therefore develop a sense of belonging and emotional wellbeing (Helliwell \& Putnam, 2004; Wellman \& Wortley, 1990). The buffer effect model posits that neighbourhood ties buffered one's psychological overloads by enhancing one's coping abilities (Kawachi \& Berkman, 2001; Lin \& Ensel, 1989; Ross \& Jang, 2000). Individuals have been shown to evaluate stressful situations and their abilities to deal with stress and depression when stressful events occur (Cohen \& Wills, 1985; Kawachi \& Berkman, 2001), and their connections with neighbours equip them with competence and confidence to deal with these stressors. Therefore, reciprocal neighbourhood ties play a positive role in alleviating individuals' anxiety and engendering their positive feelings.

Studies on the negative impacts of neighbourhood deprivation on SWB can be traced back to the Chicago School's concept of 'social disorganisation' (Park \& Burgess, 1921). Neighbourhood deprivation exerts detrimental effects on residents' SWB because it is associated with a series of social problems such as joblessness, violence, crime, and other anti-social behaviours (Sampson, Morenoff, \& GannonRowley, 2002; Wilson, 2012). People who are exposed to these social environmental stressors are more likely to feel nervous and depressed. For example, Ross and Jang (2000) found that residents in deprived neighbourhoods were more likely to suffer from anxiety and fear of crime. They also found that people living in deprived 
neighbourhoods had more difficulties than others in seeking effective supports from their neighbours, because their neighbours were short on economic and social resources.

A few studies have investigated the buffering effect of neighbourhood ties on the negative impacts of neighbourhood deprivation (Cattell, 2001; Ross \& Jang, 2000; Sampson et al., 2002; van Eijk, 2012). First, individuals benefit from reciprocity among neighbours in neighbourhoods with poor facilities and services. Second, individuals subject to poverty enhance their self-esteem and build up a sense of achievement by helping their neighbours (Wellman \& Wortley, 1990). Cattell (2001) suggested that individuals rebuild a sense of confidence in their lives based on their participation in neighbouring activities. Third, individuals display little fear of neighbourhood disorder when living in a supportive milieu. Ross and Jang (2000) argued that residents developed informal alliances with neighbours through daily mutual help, which allows them to overcome the fear of neighbourhood disorder.

\subsection{Understanding migrants' SWB and neighbourhood ties in}

\section{Chinese cities}

A great amount of literature has focused on migrants' social ties when they are away from home and live in Chinese cities temporarily (Cheng, Wang, \& Smyth, 2014; Cheung, 2014; Jin et al., 2012; Wen \& Wang, 2009; Zhu \& Lin, 2014; Huang, Dijst, \& Van Weesep,2016). While earlier literature has been centred around migrants' social ties with native-place fellows within and across neighbourhoods (Liu, Li, \& Breitung, 2012; Liu, Wang, \& Tao, 2013; Yue et al., 2013), recent studies have focused 
particularly on their social ties with neighbours (Wang, Zhang, \& Wu, 2015, 2016; Wu \& Logan, 2015). Neighbourhoods in host cities where migrants live play a key role in their social life, in part because they tend to aggregate with their native-place fellows in the same place, and also due to the fact that they have a certain level of willingness to make friends with out-group neighbours (Liu et al., 2012; Wang et al., 2016; Wu \& Logan, 2015; Yue et al., 2013).

As previously discussed, individuals' strong social ties with neighbours exert positive effects on their wellbeing. This is also true for migrants. For one thing, migrants receive both instrumental and emotional support from native-place fellows living in the same neighbourhood (Liu et al., 2012; Yue et al., 2013). For another, they gain more opportunities to integrate into host cities and achieve better SWB when developing intergroup communication and assistance with local neighbours (Wang et al., 2016; Wu \& Logan, 2015). Therefore, the first hypothesis was developed, as follows:

\section{Hypothesis 1: Migrants' SWB is positively linked to the strength of their}

\section{neighbourhood ties.}

Due to limited budget and housing choices, most migrants live in deprived neighbourhoods with poor housing qualities, dilapidated facilities, poor sanitation, high crime rates, and little access to community-level services (Chen, 2012; Huang \& Yi, 2015; Li \& Wu, 2013; Shen, 2016; Wu, 2006). Such neighbourhood-level environmental stressors raise the level of residents' stress and depression. As previously pointed out, seeking help from neighbours is sometimes an efficient means for migrants to cope with environmental stressors (Du \& Li, 2010; Liu et al., 2012). Support from 
neighbours not only prevents the occurrence of stressful events, but also curbs negative emotions arising from the exposure to stressful environments. Some studies have found a negative association between neighbourhood deprivation and migrants' SWB, yet whether neighbourhood ties play a role in mediating such an affirmatory relationship remains poorly understood. Therefore, the second hypothesis was raised, as follows:

\section{Hypothesis 2: Migrants' neighbourhood ties buffer the negative impacts of} neighbourhood deprivation.

The first two hypotheses (Hypothesis 1 and Hypothesis 2) suggest that migrants' neighbourhood ties may have both main effects and buffer effects on their SWB, but it is possible that both effects for migrants' SWB are not as strong as those for locals' SWB. This prediction was made for the following reasons. First, migrants often turn to their relatives or native-place fellows rather than intergroup neighbours for help when facing life difficulties, but such kinship and place-based ties are not necessarily bounded by neighbourhood boundaries (Liu et al., 2012; Yue et al., 2013). It is common for migrants to use their connections with relatives and friends who are left behind in the countryside or scattered across host cities to acquire social resources and deal with difficulties (Cheung, 2014; Jin et al., 2012; Liu et al., 2014).

Second, migrants usually change their residences frequently in host cities and thereby have fewer opportunities than locals to develop strong neighbourhood ties ( $\mathrm{Wu}$, 2006; Huang, Yi, Yang, \& He, 2014). Thus, migrants may have fewer and weaker neighbourhood ties than locals, especially low-income locals whose daily activities are restricted to where they live. For example, a study by Liu et al. (2012) on urban villages 
in Guangzhou indicated that compared to migrants, locals interact more frequently with their neighbours and are involved in more neighbouring activities. Further, a study by Wen et al. (2010) on neighbourhoods in Shanghai showed that neighbourhood social cohesion promoted wellbeing resources for natives rather than for migrants.

Finally, migrants may be more tolerant of environmental stressors, such as poor sanitation and facilities, that occur in the neighbourhoods where they reside due to the fact that they usually consider host cities as a place to work rather than to live, and thus have low expectations for standards of living in host cities (Li \& Wu, 2013; Xiao, Wang, Li, \& Tang, 2017; Zheng, Long, Fan, \& Gu, 2009). Therefore, it may be inferred that (1) both migrants' and locals' SWBs vary by neighbourhood ties and neighbourhood deprivation; (2) both neighbourhood ties and neighbourhood deprivation have weaker effects on migrants' SWB than on that of locals. It is thus assumed that the interaction (i.e., buffer effect) between these two psychosocial factors is also weaker for migrants. Thus, the final hypothesis was proposed:

\section{Hypothesis 3: The main effect and buffer effect of neighbourhood ties on SWB} are weaker for migrants than for locals.

\section{Data and methods}

\subsection{Data}

Guangzhou is an ideal place to undertake field studies on migrants' SWB. As the capital of Guangdong Province, the city is one of the most attractive destinations for internal migrants in China. Guangzhou accommodated 8.42 million migrants in 2015, 
which occupied more than half of the city's population (13.08 million).

The current study used data collected by a research team through a survey conducted between June and August 2015. The research team selected survey respondents through two stages. In the first stage, 23 residential communities (she qu $)^{1}$ were selected randomly from seven districts, with a multistage stratified probability proportionate to population size sampling technique (Figure 1). All of these districts (Liwan, Yuexiu, Haizhu, Tianhe, Baiyun, Panyu, and Huangpu) were located in Guangzhou's inner city areas and inner suburbs. In the second stage, 50 households from each sampled neighbourhood were randomly selected, and one household member from each household was then chosen using the Kish Grid.

We left out the outer-suburban districts in the sampling of neighbourhoods for the following reasons. First, these five districts are more like self-sustained satellite cities, as they are physically separated from the inner-city and inner-suburban districts of Guangzhou by rural areas or low-density built-up areas. Second, these five districts are partially independent from the inner-city and inner-suburban part of Guangzhou economically and socially. Third and most importantly, it is too costly to carry out field work in the outer-suburb districts, as these districts are far away from Guangzhou's city centre.

\footnotetext{
1 A community in a Chinese city is an administrative and social collective that always consists of one or more neighbourhoods adjoining each other. Residents living in the community are served and governed by the same neighbourhood committee (ju wei hui).
} 


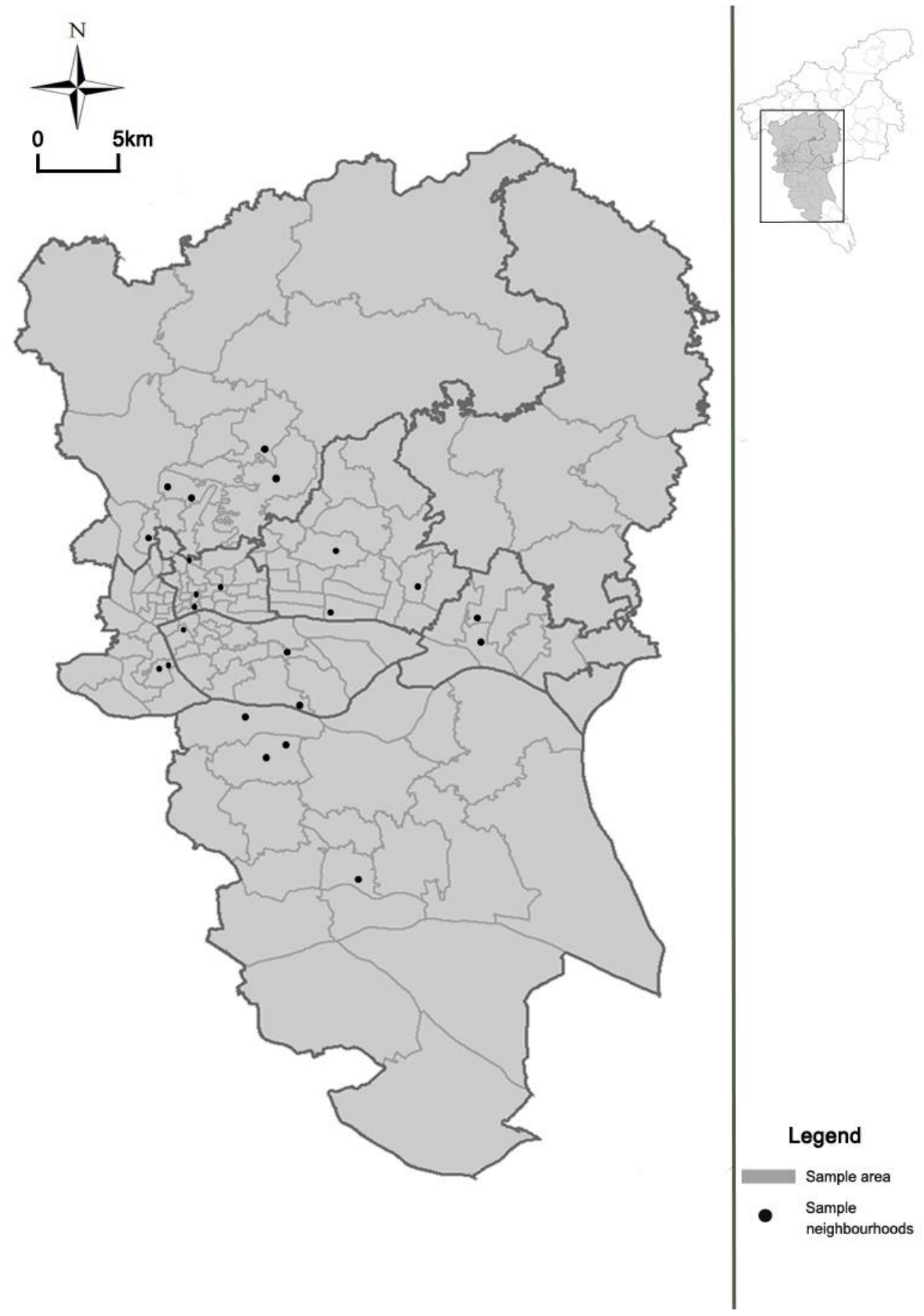

Figure 1. The location of sampled communities in Guangzhou, China

The survey yielded a total of 1,150 valid respondents, among which 467 respondents were migrants and 683 respondents were locals. The proportion of migrants in survey respondents is approximate to that in Guangzhou's population in 
$2015 .^{2}$ Given that respondents' neighbourhoods may have little influence on their SWB if they merely stay in the neighbourhood for a short period, respondents who had only lived in the sampled neighbourhood less than one year were dropped. In addition, respondents who were outside the labour force (e.g., homemakers) at the time of the survey were also dropped. The final dataset was comprised of 1,064 respondents. In addition to survey data collected by the research team, Guangzhou small-area census data from 2010 were used to construct a neighbourhood deprivation index.

\subsection{Multilevel models}

This research treated respondents' SWB as a function of their neighbouring, their neighbourhood environment, the interaction between neighbouring and neighbourhood environment, and individual controls. Given that the variable of SWB was a continuous variable, multilevel linear regressions were employed to estimate the effect of independent variables. Multilevel models are superior to single-level models due to their correct inferences and ability to isolate neighbourhood effects brought about by observable (in this case, neighbourhood deprivation) and unobservable characteristics. All continuous independent variables were grand-mean centred. We carried out the centring of independent variables in order to obtain a more interpretable intercept estimate (Enders \& Tofighi, 2007). Robust standard error was applied as a specialised estimator to correct the distributional problems caused by some transformations of data (Jackson, 2010). A variance inflation test was carried out and revealed no

\footnotetext{
2 Data from the Guangzhou Statistical Bureau (http://www.gzstats.gov.cn/tjgb/qtgb/201504/t20150430_37572.htm)
} 
multicollinearity among explanatory variables. Likelihood ratio tests were also conducted for all multilevel linear regression models and found that multilevel regressions had stronger explanatory power than single-level regressions. In this study, we simply used random intercept models instead of random coefficient models, assuming that the relationship between SWB and its neighbourhood-level predictors was fixed across neighbourhoods. In this case, there is no intercept-slope covariance and any other covariance between a pair of neighbourhood slopes. All analyses were conducted using STATA (Version 13). In the multilevel models, 426 migrants and 638 local residents at level 1 were nested within 23 neighbourhoods at level 2. Random intercepts multilevel models were specified as follows:

$$
Y_{i j}=\beta X_{i j}+\phi S_{i j}+\gamma Z_{j}+\delta S_{i j} Z_{j}+\mu_{j}+\varepsilon_{i j}
$$

Here, $Y_{i j}$ is the SWB score for person $i$ in neighbourhood $j ; X_{i j}$ denotes a set of individual controls concerning demographic characteristics, socioeconomic status, health conditions, and hukou status; $S_{i j}$ represents variables related to respondents' neighbourhood ties, including neighbourly interaction and mutual help within the neighbourhood; $Z_{j}$ represents a neighbourhood-level variable concerning the level of deprivation; $S_{i j} Z_{j}$ is the interaction between neighbourhood ties and neighbourhood deprivation; $\mu_{j}$ denotes the differences between neighbourhood $j$ 's mean and the overall mean; and $\varepsilon_{i j}$ represents individual residuals. The following sections provide a description of variables used in the models (Table 1).

Table 1 Descriptive statistics for migrants and urban residents in Guangzhou, China 


\begin{tabular}{|c|c|c|c|c|c|c|}
\hline & \multicolumn{2}{|c|}{$\begin{array}{c}\text { All samples } \\
(\mathrm{N}=1064)\end{array}$} & \multicolumn{2}{|c|}{$\begin{array}{c}\text { Migrants } \\
(\mathrm{N}=426)\end{array}$} & \multicolumn{2}{|c|}{$\begin{array}{c}\text { Local } \\
\text { residents } \\
(\mathrm{N}=638)\end{array}$} \\
\hline & Mean & (S.D.) & Mean & (S.D.) & Mean & (S.D.) \\
\hline Subjective wellbeing (5-35) & 20.9 & 5.4 & 19.9 & 5.1 & 21.5 & 5.5 \\
\hline \multicolumn{7}{|l|}{ Neighbourhood social interaction } \\
\hline Neighbourhood interaction (1-5) & 2.7 & 1.0 & 2.5 & 0.9 & 2.8 & 0.9 \\
\hline Neighbourhood mutual support (1-5) & 3.8 & 0.7 & 3.6 & 0.7 & 3.9 & 0.7 \\
\hline \multicolumn{7}{|l|}{ Neighbourhood deprivation } \\
\hline Neighbourhood deprivation index & -0.01 & 0.9 & 0.25 & 1.0 & -0.19 & 0.8 \\
\hline \multicolumn{7}{|l|}{ Hukou status (\%) } \\
\hline Guangzhou hukou holders & 60.5 & & 0.0 & & 100.0 & \\
\hline Urban hukou holders & 69.2 & & 77.1 & & 100.0 & \\
\hline \multicolumn{7}{|l|}{ Demographic characteristics } \\
\hline Age (18-70) & 40.8 & 10.9 & 37.4 & 9.6 & 43.6 & 10.7 \\
\hline \multicolumn{7}{|l|}{ Gender $(\%)$} \\
\hline Male & 52.2 & & 53.0 & & 48.0 & \\
\hline Female & 47.8 & & 47.0 & & 52.0 & \\
\hline \multicolumn{7}{|l|}{ Marital status and family organization (\%) } \\
\hline Single, divorced and widowed & 19.0 & & 17.0 & & 20.0 & \\
\hline Married and living together with family & 79.1 & & 79.0 & & 79.0 & \\
\hline Married but living apart from family & 1.9 & & 4.0 & & 1.0 & \\
\hline \multicolumn{7}{|l|}{ Socioeconomic status } \\
\hline Personal hourly income & 38.3 & 284.8 & 29.7 & 39.4 & 47.7 & 376.4 \\
\hline Social insurance in Guangzhou (0-9) & 3.4 & 2.0 & 2.6 & 2.2 & 4.0 & 1.7 \\
\hline \multicolumn{7}{|l|}{ Housing tenure $(\%)$} \\
\hline Homeowner & 55.1 & & 18.0 & & 21.0 & \\
\hline Renter & 44.9 & & 82.0 & & 79.0 & \\
\hline \multicolumn{7}{|l|}{ Education $(\%)$} \\
\hline Below high school & 31.5 & & 48.0 & & 21.0 & \\
\hline High school, college, university and above & 68.5 & & 52.0 & & 79.0 & \\
\hline \multicolumn{7}{|l|}{ Health status } \\
\hline Physical health (1-5) & 4.2 & 0.8 & 4.3 & 0.7 & 4.1 & 0.9 \\
\hline Psychological health (GHQ-12) (12-48) & 22.6 & 5.3 & 22.9 & 5.2 & 22.3 & 5.3 \\
\hline
\end{tabular}

\subsection{Measurement of variables}

\subsubsection{SWB}

The term 'subjective wellbeing' in this research is defined as one's perception of 
his/her quality of life. There are many ways to measure SWB, and social scientists continue to find new and arguably superior ways to quantify this construct (Diener, Emmons, Larsen, \& Griffin, 1985; Ryff, 1989). Analysis of respondents' SWB was based on a straightforward measure of life satisfaction. The current research applied Diener et al. (1985)'s Satisfaction with Life Scale (SWLS) to measure the level of respondents' SWB. The instrument of SWLS consists of five items, as follows: 'In most ways, my life is close to my ideal'; 'The conditions of my life are excellent'; 'I am satisfied with my life'; 'So far I have gotten the important things I want in life'; and 'If I could live my life over again, I would change almost nothing'. Respondents were asked to point out the extent to which they agree or disagree with each of the above statements, using a seven-point Likert-type scale ranging from 1 ('strongly disagree') to 7 ('strongly agree'). A higher score means a higher level of SWB. The overall score of SWB was the sum of the five scores.

\subsubsection{Neighbouring ties}

The respondents' neighbourhood ties were broken down into neighbourly interaction and mutual help among neighbours. The following question was utilised to measure neighbourly interaction: 'How often do you interact with your neighbours by greetings, daily chats, and home visits? (from 1="never" to 5="always")'. Then, the following question was used to measure mutual help among neighbours: 'To what extent do you agree with the statement that Both you and your neighbours are willing to help other residents when it is needed? (from 1="strongly disagree" to 5="strongly agree")'. To simplify the models, we converted a five-point scale into a three-point scale 
by collapsing values 1 ("never"/ "strongly disagree") and 2 ("rarely"/“disagree") into 1, changing value 3 ("sometimes"/ "undecided") to 2 , and collapsing values 4 ("very often"/ "agree") and 5 ("always"/“strongly agree") into 3, with the value of 2 treated as the reference group. Noted that most respondents chose the middle option ("sometimes"/ "undecided") in the survey. We used this strategy of grouping, because doing so would not affect substantially the distribution of data

\subsubsection{Neighbourhood deprivation}

An index of neighbourhood deprivation was developed based on four census indicators at the neighbourhood level: homeownership rates, unemployment rates, low levels of education, and low status occupation. The homeownership rate was the share of residents living on their own property in the neighbourhood. The unemployment rate was the share of residents who were unemployed. The low level of education was the share of residents with education levels equivalent to junior high school or lower. The low-status occupation included the share of residents working in the following low-end occupations: personnel of commerce, catering and service; producers of farming, forestry, animal husbandry, side-line production, and fishery; operators of production and transport equipment. Principal component analysis (PCA) was used to extract neighbourhood deprivation index from the above four indicators. We firstly standardized the data and generated the correlation matrix R. Eigenvalues and eigenvectors of factors are showed in Appendix Table 1. We then followed KaiserGuttman rule (1960) and selected only one component (component 1 with an eigenvalue of 2.681) to generate the neighbourhood deprivation index. Appendix Table 
2 reports the summary statistics of the variable of neighbourhood deprivation index.

\subsubsection{Buffering effect of neighbourhood ties}

To gauge the buffer effect of neighbourhood ties on the adverse effect of neighbourhood deprivation, interaction terms between neighbourhood deprivation and neighbourhood ties (including both neighbourly interactions and mutual assistance among neighbours) were added to the model specification.

\subsubsection{Controlled variables}

A series of controlled variables were included in the model: demographic characteristics, socioeconomic status, health conditions, and hukou status. Analysis of the respondents' health conditions was based on self-reported physical health and psychological health. Physical health was assessed with one question: 'How do you think about your health in general? (from 1="very unhealthy" to 5="very healthy")'. The measurement of self-reported psychological health was adopted from the 12-item General Health Questionnaire (GHQ-12), which estimated the severity of one's mental problems over the last few weeks. The respondents were asked to respond according to a four-point Likert-type scale. The positive items were corrected from 1 ('always') to 4 ('never'), while the negative ones ranged from 4 ('always') to 1 ('never'). The higher the score, the worse the respondent's psychological health.

\section{Results}

\subsection{Summary statistics of variables}

Table 1 displays the summary statistics of variables used in the models. As 
expected, migrants exhibit a lower level of SWB than urban residents (20.1 versus 21.4, $\mathrm{t}=4.48$ ). The average score of migrants' SWB also falls into the category of those who are slightly dissatisfied with their life. ${ }^{3}$ With respect to neighbourhood interaction, migrants interact less with their neighbours compared to local residents ( 2.5 versus 2.8 , $\mathrm{t}=-5.02$ ). In terms of neighbourly mutual support, migrants report lower ratings of mutual support among neighbours when compared to locals (3.6 versus $3.9, \mathrm{t}=-5.17$ ). Regarding neighbourhood deprivation, migrants are more likely to live in a deprived neighbourhood than locals $(0.25$ versus $-0.19, \mathrm{t}=7.58)$.

As for controlled variables, compared with local residents, migrants are on average younger, less involved in the social insurance scheme, less likely to live on their own property, and less educated. Further, they were shown to have better physical health conditions than locals. However, there is no significant difference between migrants and locals in terms of psychological health conditions.

\subsection{Descriptive analysis}

The current research explored the relationship between neighbourhood ties, neighbourhood deprivation, and individuals' SWBs (Table 2). The findings suggest that migrants with a high level of SWB tend to be those who actively interact with neighbours (19.8 versus 21.0, $\mathrm{t}=-1.826)$, and reside in mutually supportive neighbourhoods (19.2 versus $20.7, \mathrm{t}=-3.057)$ with a lower level of neighbourhood deprivation $(20.8$ versus $19.5, \mathrm{t}=2.528)$. The same observation applies to locals, whose

\footnotetext{
3 The benchmark of the SWLS is as follows: 5-9 'extremely dissatisfied', 10-14 'dissatisfied', 15-19 'slightly dissatisfied', 20 'neutral', 21-25 'slightly satisfied', 26-30 'satisfied', and 31-35 'extremely satisfied'.
} 
SWB is positively associated with their frequency of interacting with neighbours $(20.8$ versus $23.0, \mathrm{t}=-4.614)$ and their residency in supportive neighbourhoods (20.0 versus $21.9, \mathrm{t}=-4.174)$, while negatively correlated to the deprivation level of their neighbourhoods (21.9 versus $20.4, \mathrm{t}=3.601)$.

Table 2 The relationship between neighbourhood support and individuals' SWB

\begin{tabular}{lcccc}
\hline & \multicolumn{3}{c}{ Migrants' SWB } & \multicolumn{2}{c}{ Locals' SWB } \\
\cline { 2 - 5 } & Mean & S.D. & Mean & S.D. \\
\hline Neighbourhood interaction & 19.8 & 5.0 & 20.8 & 5.5 \\
Weak interaction (1 3) & 21.0 & 5.5 & 23.0 & 5.1 \\
Strong interaction (4 5) & & & & \\
Neighbourhood mutual help & 19.2 & 4.9 & 20.0 & 5.6 \\
Weak mutual help (1 3) & 20.7 & 5.2 & 21.9 & 5.4 \\
Strong mutual help (4 5) & & & & \\
Neighbourhood deprivation & 20.8 & 6.0 & 21.9 & 5.5 \\
Low-level of deprivation (-1.66 0) & 19.5 & 4.2 & 20.4 & 5.3 \\
High-level of deprivation (0.01 1.66) & & & &
\end{tabular}

\subsection{Multilevel models}

Three sets of multilevel models were initiated to estimate the main effect and buffer effect of neighbourhood ties on the SWBs of migrants, locals, and all sample members. Baseline models were used to estimate the overall effects of neighbourhood ties (Models 1a and 2a). To distinguish between the main effect and the buffer effect of neighbourhood ties, interactions between neighbourhood deprivation and neighbourhood ties ${ }^{4}$ were added to baseline models (Models $1 \mathrm{~b}$ and 2b). In Model 3, migrants and locals were grouped together and interactions were used to examine how neighbourhood ties influenced migrants' SWB and locals' SWB differently.

\footnotetext{
${ }_{4}^{4}$ The buffer effect of social ties is usually tested by using the statistic interaction between variables of stressors and variables of social ties (Cohen \& Wills, 1985; Lin \& Ensel, 1989; Ross \& Jang, 2000).
} 
Table 3 illustrates the results of five multilevel models. As expected, respondents' SWB was negatively associated with the neighbourhood deprivation index of their neighbourhoods. More specifically, a one-point increase in the neighbourhood deprivation index causes a decrease in the score of SWB by 0.739 for migrants (Model 1a), by 0.965 for local residents (Model 2a), and by 0.810 for all sample members (Model 3). As for the overall effect of neighbourhood ties, Model 1a shows that migrants' SWB was positively related to the frequency of interacting with their neighbours and their perceived level of mutual help among neighbours. Specifically, migrants who interacted frequently with their neighbours displayed higher SWB than other migrants by 1.090 points, and migrants who perceived a high level of mutual help among neighbours had higher SWB than other migrants by 0.613 points (Model 1a). This finding verifies Hypothesis 1. Then, the buffering effects of neighbourhood ties were gauged by taking them apart from main effects. Neighbourhood ties appear to exert a positive main effect on migrants' SWB. However, inconsistent with Hypothesis 2, no evidence has shown that the buffering effect hypothesis applies to migrants (Model 1b).

Although migrants' neighbourhood ties exerted a positive overall effect on their SWB, as suggested by Hypothesis 3, such an effect was not as strong as that of locals. Locals who interacted frequently with their neighbours displayed higher SWB than other locals by 1.791 points, and those who perceived a high level of mutual help among neighbours displayed higher SWB than those who perceived a medium and a low level of mutual help by 1.603 points and 4.505 points, respectively (Model 2a). Results from 
Model 3 also confirm this finding: interaction effects between Guangzhou hukou status and neighbourly interaction (or mutual assistance among neighbours) are significant and positive. This indicates a stronger positive effect of both neighbourly interaction and mutual help within neighbourhoods on SWB for locals than for migrants.

For the buffering effects, there is no evidence indicating that migrants' neighbourhood ties lessen the stress arising from living in deprived neighbourhoods. However, the buffer effect hypothesis applies to locals' SWB, as the interaction effect between neighbourhood deprivation and mutual help among neighbours is positive and significant at the ten percent level. This indicates that the adverse effects of neighbourhood deprivation on locals' SWB can be alleviated by real support from neighbours, but not by superficial interactions with neighbours. As such, Hypothesis 3 of this research has been fully confirmed. 
Table 3 Multilevel modelling on respondents' subjective wellbeing in Guangzhou

\section{Independent variables}

Neighbourhood deprivation index (NDI) Neighbourhood interaction

(ref: medium)

$$
\begin{aligned}
& \text { High } \\
& \text { Low }
\end{aligned}
$$

Neighbourhood mutual help

(ref: medium)

$$
\begin{aligned}
& \text { High } \\
& \text { Low }
\end{aligned}
$$

Interaction effects

NDI X High-level of neighbourhood interaction

NDI X Low-level of neighbourhood interaction

NDI X High-level of neighbourhood help NDI X Low-level of neighbourhood help Hukou status and its interaction effects Guangzhou hukou (ref: non-Guangzhou hukou status)

Guangzhou hukou X High-level of

\begin{tabular}{|c|c|c|c|c|c|c|c|c|c|}
\hline \multicolumn{4}{|c|}{ Migrants } & \multicolumn{4}{|c|}{ Locals } & \multirow{2}{*}{\multicolumn{2}{|c|}{$\begin{array}{c}\text { Overall } \\
\text { Model } 3\end{array}$}} \\
\hline \multicolumn{2}{|c|}{ Model 1a } & \multicolumn{2}{|c|}{ Model 1b } & \multicolumn{2}{|c|}{ Model 2a } & \multicolumn{2}{|c|}{ Model 2b } & & \\
\hline Estimate & $\begin{array}{c}\text { Robust } \\
\text { S.E. }\end{array}$ & Estimate & $\begin{array}{c}\text { Robust } \\
\text { S.E. }\end{array}$ & Estimate & $\begin{array}{c}\text { Robust } \\
\text { S.E. }\end{array}$ & Estimate & $\begin{array}{c}\text { Robust } \\
\text { S.E. }\end{array}$ & Estimate & $\begin{array}{c}\text { Robust } \\
\text { S.E. }\end{array}$ \\
\hline$-0.739 * *$ & $(0.375)$ & $-0.707^{*}$ & $(0.372)$ & $-0.965^{* *}$ & $(0.405)$ & $-1.447 * * *$ & $(0.511)$ & $-0.810^{* *}$ & $(0.358)$ \\
\hline $1.090^{*}$ & $(0.610)$ & $1.147 *$ & $(0.654)$ & $1.791 * * *$ & $(0.322)$ & $1.782 * * *$ & $(0.343)$ & $1.168 *$ & $(0.651)$ \\
\hline-0.003 & $(0.527)$ & 0.108 & $(0.578)$ & -0.957 & $(0.640)$ & -1.077 & $(0.757)$ & 0.026 & $(0.520)$ \\
\hline $0.613 * *$ & $(0.304)$ & $0.542 *$ & $(0.290)$ & $1.603 * * *$ & $(0.505)$ & $1.725 * * *$ & $(0.461)$ & $0.516^{*}$ & $(0.302)$ \\
\hline-0.850 & $(0.911)$ & -0.718 & $(1.588)$ & $-2.902 * * *$ & $(0.997)$ & $-2.475 * * *$ & $(0.913)$ & -0.832 & $(0.903)$ \\
\hline
\end{tabular}
neighbourhood interaction

Guangzhou hukou X Low-level of neighbourhood interaction

$\begin{array}{llll}-0.111 & (0.452) & 0.684 * * & (0.326) \\ -0.388 & (0.439) & -0.472 & (0.751) \\ 0.280 & (0.307) & 0.934 * & (0.553) \\ -0.001 & (1.022) & -1.380 * * * & (0.479)\end{array}$

$\begin{array}{ll}-0.471 & (0.564) \\ 0.661 * * & (0.307) \\ -0.802 & (0.797)\end{array}$




\section{Controlled variables}

\section{Demographic characteristics}

Age

Sex (ref: male)

0.034

(0.021) $\quad 0.033$

$(0.022) \quad 0.019$

(0.023) $\quad 0.015$

$(0.022) \quad 0.020$

$(0.015)$

Marital status and family organisation (ref:

Married and living together with spouse)

Single, divorced or widowed

$-0.383$

(0.501) $\quad-0.422$

(0.504) $0.948 * *$

(0.377) $0.971^{* *}$

(0.386) $\quad 0.378$

$(0.267)$

Married but living apart from family

$\begin{array}{lllllllllll}0.750 & (0.856) & 0.733 & (0.867) & 0.176 & (0.526) & 0.073 & (0.528) & 0.287 & (0.524) \\ 0.026 & (1.294) & -0.007 & (1.298) & 0.148 & (2.529) & -0.250 & (2.456) & 0.114 & (1.255) \\ & & & & & & & & & \\ 0.922^{* * *} & (0.316) & 0.904 * * * & (0.307) & 1.053^{* * *} & (0.350) & 1.074 * * * & (0.348) & 1.021^{* * *} & (0.256) \\ -0.080 & (0.105) & -0.080 & (0.104) & 0.266^{*} & (0.142) & 0.253^{*} & (0.142) & 0.058 & (0.088) \\ 1.537 * * & (0.722) & 1.587 * * & (0.683) & 1.598^{* * *} & (0.592) & 1.689^{* * *} & (0.595) & 1.462 * * * & (0.439) \\ & & & & & & & & & & \\ & & & & & & & & & \\ 0.304 & (0.491) & 0.288 & (0.496) & 0.332 & (0.511) & 0.271 & (0.508) & 0.336 & (0.347) \\ 1.227 * & (0.634) & 1.249 * * & (0.634) & 0.799 & (0.606) & 0.761 & (0.594) & 1.112^{* *} & (0.495) \\ -0.074 & (0.053) & -0.072 & (0.054) & -0.062 & (0.051) & -0.057 & (0.048) & -0.067 * & (0.038) \\ 15.937 * * * & (1.960) & 15.993^{* * *} & (2.004) & 13.836^{* * *} & (2.289) & 13.834 * * * & (2.281) & 15.468 * * * & (1.444) \\ 20.642^{* * *} & (2.952) & 20.601 * * * & (2.913) & 22.785^{* * *} & (1.718) & 22.675^{* * *} & (1.808) & 22.249 * * * & (1.944) \\ 1.691 * & (0.677) & 1.865 * & (0.897) & 1.353^{*} & (0.578) & 1.922^{*} & (0.755) & 1.570^{*} & (0.675) \\ 426 & & 426 & & 638 & & 638 & & 1064 & \end{array}$

Personal hourly income (natural log)

Social insurance in Guangzhou

Housing tenure

Education attainment (ref: junior high school or below)

Senior high school or above

Self-reported health conditions

Physical health

Psychological health

Constant

Within area variances

Between area variances

$-1258.9$

$-1260.4$

$-1911.6$

$-1909.2$

$-3174.1$

Notes: Numbers in parentheses are robust standard errors. All continuous independent variables are grand-mean centred. *, **and *** denote statistical significance at the $10 \%, 5 \%$, and $1 \%$ levels, respectively. 


\section{Conclusion and discussion}

Existing literature on migrants' SWB in Chinese cities has highlighted the crucial role of social ties, yet the pathways (i.e., processes) by which social ties influence their SWB remain poorly understood. Using Guangzhou survey data and multilevel linear regressions, this paper examines the extent to and ways in which migrants' social ties with neighbours enhance their SWB, with a particular focus on the distinction between the main effects and buffering effects of their neighbourhood ties. Results from multilevel models reveal that neighbourhood ties enhance migrants' SWB in a direct manner, but no evidence shows that neighbourhood ties buffer the negative impacts of neighbourhood deprivation. Results also illustrated that the association between neighbourhood ties and SWB is stronger for locals than for migrants, and that the buffering effects of neighbourhood ties exist for locals' SWB.

While earlier studies have reported the positive role of residential neighbourhoods in migrants' daily activities and social lives (Liu et al., 2012; Wang et al., 2015; Wu \& Logan, 2015), the current study makes a further step to investigate the pathways by which migrants' connections with neighbours enhance their SWB. Specifically, the current research distinguishes two pathways through which neighbourhood ties influence migrants' SWB: the main effects and buffer effects.

The findings challenge the assumption that migrants' neighbourhood ties play a role in buffering stress associated with deprived neighbourhood environments. There are several explanations for this observation. First, migrants' social ties are less bound by neighbourhood boundaries than those of locals. Second, compared to locals, 
migrants have fewer opportunities to develop strong neighbourhood ties. Third, migrants' SWB is less subject to stressors associated with neighbourhood deprivation, as most migrants have low expectations on the standard of living in host cities. Further analysis is required to examine the buffering effect of other social ties (e.g., social ties with relatives and friends living outside the neighbourhood and in the hometown) on the adverse effect of neighbourhood deprivation.

This article makes conceptual and empirical contributions to the understanding of migrants' SWB in Chinese cities. Conceptually, the current research incorporates the buffering effect of neighbourhood ties on the negative impact of neighbourhood deprivation into the analytical framework of migrants' SWB. This paper also contributes to SWB research by revealing impacts from the identity of disadvantaged social group on SWB. Empirically, this study uses the interaction terms between neighbourhood ties and neighbourhood deprivation to capture the buffering effect.

Our findings have some policy implications. First, policy efforts should be made to build a more supportive and cohesive neighbourhood, as our findings have suggested that peoples' social ties with neighbours enhance their SWB. Second, in order to enhance migrants' SWB, policymakers are advised to reassess the current marketized and discriminatory housing policies leading to the concentration of migrants in deprived neighbourhoods (Li \& Wu, 2008; Chen, 2016). Third, although this study has shown no evidence of buffering effect of neighbourhood ties for migrants, it does not mean that there is no such effect for migrants in the future, especially when most migrants have no intention to return and consider the host city as a place of permanent settlement. 
Therefore, enabling migrants to build localised social ties will be a good way to alleviate the negative effect of area deprivation in polarising Chinese cities.

Despite these contributions, some shortcomings of this study should be noted. First, there may be measurement errors in the independent variables of the models, as only two specific indicators (neighbourly interaction and mutual help among neighbours) were used rather than comprehensive indicators to measure informational, instrumental, and emotional support from neighbours. Second, there could be a downward bias in the estimates of buffering effects of neighbourhood ties, as the extent to which neighbourhood ties buffered the negative impact of other types of life stress (e.g., work stress) were not measured. Third, the estimates from regressions may be biased due to the presence of unobserved individual heterogeneity. For example, the models in the current study did not take into account individuals' personality traits, which are possibly linked to their SWB. Fourth, it might be possible that the sample size for this study was not large enough to detect the buffering effect of migrants' neighbourhood ties on their SWB. To address this problem, researchers would be advised to verify the findings of this research based on data from large-scale nationally representative survey.

\section{Acknowledgement}

The authors would like to thank the editor and the anonymous reviewers for their helpful and constructive comments that greatly contributed to improving the paper. This work was supported by the National Natural Science Foundation of China 
[41320104001, 41422103, 41501151]; the UK Economic and Social Research Council

(ESRC)/Department for International Development (DFID) project [RES-167-25-

0448]; ESRC project [ES/N015185/1]; and the China Scholarship Council.

\section{Appendix}

Appendix Table 1. Principal component analysis of factors contributing to neighbourhood deprivation index

\begin{tabular}{lcccc}
\hline & \multicolumn{4}{c}{ Eigenvectors } \\
\cline { 2 - 4 } Factors & $\mathrm{V} 1$ & $\mathrm{~V} 2$ & $\mathrm{~V} 3$ & $\mathrm{~V} 4$ \\
\hline Low levels of education & 0.581 & -0.125 & 0.01 & 0.804 \\
Homeownership rates & -0.572 & 0.035 & 0.709 & 0.411 \\
Low status occupation & 0.573 & 0.026 & 0.704 & -0.419 \\
Unemployment rates & 0.379 & 0.691 & -0.042 & 0.098 \\
Eigenvalue & 2.681 & 0.799 & 0.201 & 0.119 \\
Proportion of variance explained (\%) & 70.6 & 21.0 & 5.3 & 3.1 \\
\hline
\end{tabular}

Appendix Table 2 Summary statistics of neighbourhood deprivation index across 23

neighbourhoods

\begin{tabular}{lc}
\hline & Neighbourhood deprivation index \\
\hline Mean & 0.000 \\
Standard deviation & 0.959 \\
Minimum & -1.650 \\
Maximum & 1.650 \\
\hline
\end{tabular}

\section{References}

Carmel, S. (2001). Subjective Evaluation of Health in Old Age: The Role of Immigration Status and Social Environment. The International Journal of Aging and Human Development, 53(2), 91-105.

Cattell, V. (2001). Poor people, poor places, and poor health: the mediating role of social 
networks and social capital. Social Science \& Medicine, 52(10), 1501-1516.

Chen, G. (2012). Housing the urban poor in post-reform China: Some empirical evidence from the city of Nanjing. Cities, 29(4), 252-263.

Chan, K. W. \& Zhang, L. (1999). The Hukou system and rural-urban migration in China: Processes and changes. China Quarterly, 818-855.

Chen, Z. H. \& Davey, G. (2008). Happiness and Subjective Wellbeing in Mainland China. Journal of Happiness Studies, 9(4), 589-600.

Cheng, Z. M., Wang, H. N. \& Smyth, R.(2014). Happiness and job satisfaction in urban China: A comparative study of two generations of migrants and urban locals. Urban Studies, 51(10), 2160-2184.

Cheung, N. W. T. (2014). Social stress, locality of social ties and mental well-being: The case of rural migrant adolescents in urban China. Health \& Place, 27, 142-154.

Chen, G. (2016). The heterogeneity of housing-tenure choice in urban China: A case study based in Guangzhou. Urban Studies, 53(5), 957-977.

Cohen, S. \& Wills, T. A. (1985). Stress, social support, and the buffering hypothesis. Psychological Bulletin, 98(2), 310-357.

Diener, E., Emmons, R. A., Larsen, R. J. \& Griffin, S. (1985). The Satisfaction with Life Scale. Journal of Personality Assessment, 49 (1), 71-75.

Du, H. \& Li, S.-M. (2010). Migrants, Urban Villages, and Community Sentiments: a Case Study of Guangzhou, China. Asian Geographer, 27(1-2), 93-108.

Enders, C. K., \& Tofighi, D. (2007). Centering predictor variables in cross-sectional multilevel models: a new look at an old issue. Psychological methods, 12(2), 121-138.

Fan, C. C. (2002). The elite, the natives, and the outsiders: Migration and labor market segmentation in urban China. Annals of the Association of American Geographers, 92(1), 103-124.

Helliwell, J. F. \& Putnam, R. D. (2004). The social context of well-being. Philosophical Transactions of The Royal Society B Biological Sciences, 359(1449), 1435-1446.

Herrero, J., Fuente, A. \& Gracia, E. (2011). Covariates of Subjective well-being among Latin American immigrants in Spain: the role of social integration in the community. Journal of Community Psychology, 39(7), 761-775.

Huang, Y. \& Yi, C. (2015). Invisible migrant enclaves in Chinese cities: Underground living in Beijing, China. Urban Studies, 52 (15), 2948-2973.

Huang, Y., Yi, C., Yang, Y. \& He, X. (2014). Mobility, housing inequality and residential differentiation in transitional urban China. In: HUANG, Y. \& LI, S.-M. (eds.) Housing Inequality in China (pp.37-63). London: Routledge

Huang, X., Dijst, M., \& Van Weesep, J. (2016). Social networks of rural-urban migrants after residential relocation: evidence from Yangzhou, a medium-sized Chinese city. Housing Studies, 1-25.

Hui, E. C. M., Yu, K. H. \& Ye, Y. (2014). Housing Preferences of Temporary Migrants in Urban China in the wake of Gradual Hukou Reform: A Case Study of Shenzhen. International Journal of Urban and Regional Research, 38(4), 1384-1398.

Jin, L., Wen, M., Fan, J. X. \& Wang, G. X. (2012). Trans-local ties, local ties and psychological well-being among rural-to-urban migrants in Shanghai. Social Science \& Medicine, 75(2), 288-296. 
Jackson, D. L. (2010). Reporting results of latent growth modeling and multilevel modeling analyses: Some recommendations for rehabilitation psychology. Rehabilitation Psychology, 55(3), 272-285.

Kawachi, I. \& Berkman, L. F. (2001). Social ties and mental health. Journal of Urban health, 78(3), 458-467.

Kaiser, H. F. (1960). The application of electronic computers to factor analysis. Educational and psychological measurement, 20(1), 141-151.

Li, B. (2006). Floating Population or Urban Citizens? Status, Social Provision and Circumstances of Rural-Urban Migrants in China. Social Policy \& Administration, 40(2), 174-195.

Li, Z. \& Wu, F. (2013). Residential Satisfaction in China's Informal Settlements: A Case Study of Beijing, Shanghai, and Guangzhou. Urban Geography, 34(7), 923-949.

Lin, N. \& Ensel, W. M. (1989). Life Stress and Health: Stressors and Resources. American Sociological Review, 54 (3), 382-399.

Liu, Y., Li, Z. \& Breitung, W. (2012). The social networks of new-generation migrants in China's urbanized villages: A case study of Guangzhou. Habitat International, 36, $192-$ 200.

Liu, Y., Li, Z., Liu, Y. \& Chen, H. (2014). Growth of rural migrant enclaves in Guangzhou, China: Agency, everyday practice and social mobility. Urban Studies, 52(16).

Liu, Y., Zhang, F., Wu, F., Liu, Y. \& Li, Z. (2017). The subjective wellbeing of migrants in Guangzhou, China: The impacts of the social and physical environment. Cities, 60, Part A, 333-342.

Liu, Z., Wang, Y. \& Tao, R. (2013). Social Capital and Migrant Housing Experiences in Urban China: A Structural Equation Modeling Analysis. Housing Studies, 28(8), 1155-1174.

Li, Z., \& Wu, F. (2008). Tenure - based residential segregation in post - reform Chinese cities: a case study of Shanghai. Transactions of the Institute of British Geographers, 33(3), 404-419.

Ma, L. J. \& Xiang, B. (1998). Native place, migration and the emergence of peasant enclaves in Beijing. The China Quarterly, 155(155), 546-581.

Mitra, A. (2010). Migration, livelihood and well-being: evidence from Indian City Slums. Urban Studies,47(7),1371-1390.

Niu, X., Xu, W., Liu, Y., Wang, G. \& Klein, K. (2016). Job-Search Channels of Migrant Workers in Large Chinese Cities: A Case Study of Shanghai. China Review, 16, 69-91.

Park, R. E. \& Burgess, E. W. (1921). Introduction to the Science of Sociology, University of Chicago Press Chicago.

Ross, C. E. \& Jang, S. J. (2000). Neighborhood disorder, fear, and mistrust: The buffering role of social ties with neighbors. American journal of community psychology, 28 (4), 401420.

Ross, C. E., Reynolds, J. R. \& Geis, K. J. (2000). The contingent meaning of neighborhood stability for residents' psychological well-being. American Sociological Review, 65(4), 581-597.

Ryff, C. D. (1989). Happiness is everything, or is it? Explorations on the meaning of psychological well-being. Journal of personality and social psychology, 57, 1069-1081.

Sampson, R. J. (2003). The Neighborhood Context of Well Being. Perspectives in Biology and 
Medicine, 46, S53-S73.

Sampson, R. J., Morenoff, J. D. \& Gannon-rowley, T. (2002). Assessing" neighborhood effects": Social processes and new directions in research. Annual review of sociology, 28, 443478.

Shen, J. (2016). Stuck in the suburbs? Socio-spatial exclusion of migrants in Shanghai. Cities. Van Eijk, G. (2012). Good Neighbours in Bad Neighbourhoods: Narratives of Dissociation and Practices of Neighbouring in a 'Problem' Place. Urban Studies, 49 (14), 3009-3026.

Wang, W. W. \& Fan, C. C. (2012). Migrant Workers' Integration in Urban China: Experiences in Employment, Social Adaptation, and Self-Identity. Eurasian Geography and Economics, 53(6), 731-749.

Wang, Z., Zhang, F. \& Wu, F. (2015). Social Trust Between Rural Migrants and Urban Locals in China-Exploring the Effects of Residential Diversity and Neighbourhood Deprivation. Population, Space and Place.

Wang, Z., Zhang, F. \& Wu, F. (2016). Intergroup neighbouring in urban China: Implications for the social integration of migrants. Urban Studies, 53 (4), 651-668.

Wellman, B. \& Wortley, S. (1990). Different Strokes from Different Folks: Community Ties and Social Support. American Journal of Sociology, 96 (3), 558-588.

Wen, M., Fan, J., Jin, L. \& Wang, G. (2010). Neighborhood effects on health among migrants and natives in Shanghai, China. Health \& Place, 16 (3), 452-460.

Wen, M. \& Wang, G. 2009. Demographic, Psychological, and Social Environmental Factors of Loneliness and Satisfaction among Rural-to-Urban Migrants in Shanghai, China. International Journal of Comparative Sociology, 50 (2), 155-182.

Wilson, W. J. (2012). The truly disadvantaged: The inner city, the underclass, and public policy, University of Chicago Press.

Wu, F. (2016). Housing in Chinese Urban Villages: The Dwellers, Conditions and Tenancy Informality. Housing Studies, 31, 852-870.

Wu, F. \& Logan, J. (2015). Do rural migrants 'float'in urban China? Neighbouring and neighbourhood sentiment in Beijing. Urban Studies,53, 2973-2990.

Wu, W. (2004). Sources of Migrant Housing Disadvantage in Urban China. Environment and Planning A, 36(7), 1285-1304.

Wu, W. (2006). Migrant Intra-urban Residential Mobility in Urban China. Housing Studies, 21(5), $745-765$.

Xiao, Y., Wang, Z., Li, Z. \& Tang, Z. (2017). An assessment of urban park access in Shanghai - Implications for the social equity in urban China. Landscape and Urban Planning, 157, 383-393.

Yue, Z., Li, S., Jin, X. \& Feldman, M. W. (2013). The Role of Social Networks in the Integration of Chinese Rural-Urban Migrants: A Migrant-Resident Tie Perspective. Urban Studies, 50(9), 1704-1723.

Zhang, L. (2012). Economic Migration and Urban Citizenship in China: The Role of Points Systems. Population and Development Review, 38(3), 503-533.

Zhang, L. \& Tao, L. (2012). Barriers to the acquisition of urban hukou in Chinese cities. Environment and Planning-Part A, 44(12), 2883.

Zheng, S., Long, F., Fan, C. C. \& Gu, Y. (2009). Urban Villages in China: A 2008 Survey of Migrant Settlements in Beijing. Eurasian Geography and Economics, 50 (4), 425-446. 
Zhu, Y. \& Lin, L. (2014). Continuity and change in the transition from the first to the second generation of migrants in China: Insights from a survey in Fujian. Habitat International, $42,147-154$. 